\title{
Classical sequences revisited with permutations avoiding dotted pattern
}

\author{
Jean-Luc Baril \\ LE2I UMR-CNRS 5158, Université de Bourgogne \\ B.P. 47 870, 21078 DIJON-Cedex France \\ barjl@u-bourgogne.fr
}

Submitted: Mar 13, 2011; Accepted: Jul 21, 2011; Published: Sep 9, 2011

Mathematics Subject Classification: 05A05

\begin{abstract}
Inspired by the definition of the barred pattern-avoiding permutation, we introduce the new concept of dotted pattern for permutations. We investigate permutations classes avoiding dotted patterns of length at most 3, possibly along with other classical patterns. We deduce some enumerating results which allow us to exhibit new families of permutations counted by the classical sequences: $2^{n}$, Catalan, Motzkin, Pell, Fibonacci, Fine, Riordan, Padovan, Eulerian.
\end{abstract}

Keywords: pattern avoiding permutation class; dotted pattern

\section{Introduction and notation}

Let $S_{n}$ be the set of permutations on $[n]=\{1,2, \ldots, n\}$, i.e., all one-to-one correspondences from $[n]$ into itself. We represent a permutation $\pi \in S_{n}$ in one-line notation $\pi=\pi(1) \pi(2) \ldots \pi(n)$. For instance the identity permutation $i d_{n}$ of length $n$ will be written $12 \ldots n$. A permutation $\pi \in S_{n}$ avoids the pattern $\tau \in S_{k}$ if and only if there does not exist a sequence of indices $1 \leq i_{1}<i_{2}<\cdots<i_{k} \leq n$ such that $\pi\left(i_{1}\right) \pi\left(i_{2}\right) \ldots \pi\left(i_{k}\right)$ is order-isomorphic to $\tau$ (see $[13,15]$ ). We denote by $S_{n}(\tau)$ the set of permutations of $S_{n}$ avoiding the pattern $\tau$. For example, if $\tau=321$ then $34125 \in S_{5}(\tau)$ while $43512 \notin S_{5}(\tau)$. Many classical sequences in combinatorics appear as the cardinality of pattern-avoiding permutation classes. A large number of these results were firstly obtained by West and Knuth $[9,13,15,17,18]$ (see the surveys of Kitaev and Mansour $[8,10]$ ).

In the literature we can also find the concept of barred pattern (see [7, 16], [11] for a systematic study of these patterns and $[2,5,15,19]$ for some applications). Let $\tau$ be a permutation in $S_{k}$ and $b=b_{1} \ldots b_{k} \in\{0,1\}^{k}$. The barred pattern $\bar{\tau}$ is a permutation in 
$S_{k}$ obtained from $\tau$ by copying the entries of $\tau$ and by putting a bar over $\tau_{i}$ if and only if $b_{i}=1$. For example, if $\tau=312$ and $b=001$ then $\bar{\tau}=31 \overline{2}$. Let $r$ be the number of barred entries in $\bar{\tau}$ (or equivalently the number of ones in $b$ ). We denote the permutation on $[k-r]$ made up of the $(k-r)$ unbarred elements of $\bar{\tau}$ by $\hat{\tau}$, rewritten to be a permutation on $[k-r]$. Obviously $\tau$ contains the pattern $\hat{\tau}$ and we will say that $\tau$ extends the pattern $\hat{\tau}$ according to the binary string $b$ (the positions of the extended entries are the same as the positions of $1 \mathrm{~s}$ in $b$ ). The permutation $\pi \in S_{n}$ avoids the pattern $\bar{\tau}$ if and only if each occurrence of the pattern $\hat{\tau}$ in $\pi$ can be extended into an occurrence of the pattern $\tau$ in $\pi$ according to $b$. For example, if $\bar{\tau}=21 \overline{34} 65$ then $b=001100,3124576 \in S_{7}(\bar{\tau})$ and $3142576 \notin S_{7}(\bar{\tau})$. Moreover we will say that $\pi \in S_{n}$ weakly avoids the pattern $\bar{\tau}$ if and only if each occurrence of the pattern $\hat{\tau}$ in $\pi$ can be extended into an occurrence of the pattern $\tau$ in $\pi$ (without considering the string $b$ ). Let $S_{n}^{w}(\bar{\tau})$ be the set of $n$-length permutations that weakly avoid $\bar{\tau}$. For example, if $\bar{\tau}=12 \overline{3}$ then $1243 \in S_{4}^{w}(\bar{\tau})$ when $1243 \notin S_{4}(\bar{\tau})$ since the sequence 24 cannot be extended into a pattern 123 according to $b=001$. By definition the weak avoidance is less restrictive than the classical avoidance: $S_{n}(\bar{\tau}) \subset S_{n}^{w}(\bar{\tau})$. Surprisingly and to my knowledge, the weak avoidance has not been studied in the literature. One reason could be that the weak avoidance on permutation classes does not provide the classical integer sequences $\left(2^{n}\right.$, Fibonacci, Motzkin, Catalan, ...). However using a variation of this concept we retrieve most of these sequences.

Now we define a dotted pattern $\dot{\tau}$ which adds dots over some entries $\tau_{i}, i \in I \subset[k]$, of $\tau \in S_{k}$. For each $j \notin I$, let $\bar{\tau}^{j}$ be the barred permutation of length $k$ obtained from $\tau$ by adding a bar over the entry $\tau_{j}$. We will say that a permutation $\pi$ avoids $\dot{\tau}$ if and only if $\pi$ weakly avoids $\bar{\tau}^{j}$ for all $j \notin I$. For example, if $\dot{\tau}=13 \dot{2}$ then $\bar{\tau}^{1}=\overline{1} 32, \bar{\tau}^{2}=1 \overline{3} 2$ and $S_{5}(\dot{\tau})=\{15243,15432,13254,13542\}$; if $\dot{\tau}=23 \dot{1}$ then $\bar{\tau}^{1}=\overline{2} 31, \bar{\tau}^{2}=2 \overline{3} 1$ and $S_{3}(\dot{\tau})=\{123,231\}$. Less formally, if $\tau^{j}$ is the pattern $\bar{\tau}^{j}$ without its barred entry then $\pi$ avoids $\dot{\tau}$ if and only if each pattern $\tau^{j}(j \notin I)$ can be extended into the pattern $\tau$ in $\pi$ (without restriction for the position of the extended entry). A particular case of this definition was studied in [3].

By convention we set $S_{n}(\dot{\tau})=S_{n}$ in the case where all entries of $\dot{\tau}$ are dotted. In the sequel we consider that the pattern $\dot{\tau}$ contains at least one dotted entry and one undotted entry. More generally we always have

$$
\begin{gathered}
S_{n}(\tau) \subseteq S_{n}\left(\tau^{j}\right) \subseteq S_{n}(\dot{\tau}) \subseteq S_{n}^{w}\left(\bar{\tau}^{j}\right) \text { for all } j \notin I, \text { and } \\
S_{n}(\dot{\tau})=\bigcap_{j \notin I} S_{n}^{w}\left(\bar{\tau}^{j}\right) .
\end{gathered}
$$

We say that $\pi$ contains $\dot{\tau}$ if $\pi$ does not avoid $\dot{\tau}$.

Moreover the Wilf equivalences are also valid: i.e., if $\delta=\dot{\tau}$ then we have

$$
\left|S_{n}(\delta)\right|=\left|S_{n}\left(\delta^{r}\right)\right|=\left|S_{n}\left(\delta^{c}\right)\right|=\left|S_{n}\left(\delta^{-1}\right)\right|
$$

where $\delta^{r}$ denotes the reverse of $\delta$ where the positions of dots are also reversed (i.e., for $i \in I$, the position $i$ is replaced with $n-i+1), \delta^{c}$ denotes the complement of $\delta$ where the 
positions of dots are preserved, and $\delta^{-1}$ denotes the inverse of $\delta$ where the dots are over the entries $i$ for $i \in I$.

In this paper, we present some enumeration results for some classes of permutations avoiding dotted patterns. More precisely, Section 2 presents some basic general lemmas concerning sets of permutations avoiding dotted patterns. Section 3 investigates exhaustively the sets of permutations avoiding one pattern $2 \dot{i}$ possibly along with one classical pattern of length 3. Section 4 deals with dotted patterns of length 3. Several open questions are given about the enumeration of one dotted patterns of length 3 . However we present others enumeration results for the case where the dotted pattern is associated with one or more other classical patterns. This study allows us to exhibit several new families of avoiding permutations that are enumerated by the well known sequences: Catalan, Motzkin, Fibonacci, Riordan, binary, Fine, Padovan, Eulerian.

\section{Some general results}

In this section we provide some general lemmas about sets of permutations avoiding dotted patterns. As defined in Section 1, we consider a dotted pattern $\dot{\tau}$ and the permutation $\tau \in S_{k}$ obtained from $\dot{\tau}$ by deleting its dots. If $I \subset[k]$ is the set of dotted positions in $\dot{\tau}$ then we also need patterns $\bar{\tau}^{j}$ and $\tau^{j}$ for $j \notin I$ (see Section 1 for these definitions).

Lemma 1 Let $\dot{\tau}$ be a dotted pattern with only one undotted entry. Then we have

$$
S_{n}(\dot{\tau})=S_{n}^{w}(\bar{\tau})
$$

where the pattern $\bar{\tau}$ is obtained from $\tau$ by adding a bar over the entry corresponding to the only one undotted entry of $\dot{\tau}$.

Proof. Here only one position $j$ is undotted in $\dot{\tau}$. So we have $I=[k] \backslash\{j\}$ which induces only one barred pattern $\bar{\tau}^{j}$. By definition, $\pi$ avoids $\dot{\tau}$ if and only if $\pi$ weakly avoids $\bar{\tau}^{j}=\bar{\tau}$ which achieves the proof.

Lemma 2 Let $\dot{\tau}$ be a dotted pattern such that all patterns $\tau^{j}, j \notin I$, are identical. Then $S_{n}(\dot{\tau})=S_{n}^{w}\left(\bar{\tau}^{j}\right)$ for each $j \notin I$.

Proof. Let $j$ and $k$ be two integers that do not lie in $I$. Recall that $\tau^{j}$ (resp. $\tau^{k}$ ) is the permutation obtained from $\bar{\tau}^{j}$ (resp. $\bar{\tau}^{k}$ ) by deleting its barred entries. By definition, a permutation $\pi$ avoids $\dot{\tau}$ if and only if each pattern $\tau^{j}(j \notin I)$ can be extended into the pattern $\tau$ in $\pi$. Therefore we directly deduce $S_{n}(\dot{\tau})=S_{n}^{w}\left(\bar{\tau}^{j}\right)$ for each $j \notin I$.

Now Lemma 2 induces the following result:

Lemma 3 Let $\dot{\tau}=k(k+1) \ldots n \dot{\delta}$ be a dotted pattern such that $\dot{\delta}$ is obtained from $\delta \in S_{k-1}$ by dotting all its entries. Then $S_{n}(\dot{\tau})=S_{n}^{w}(\bar{k}(k+1) \ldots n \delta)$.

In the remainder of this study, we focus on the enumeration of permutation classes avoiding dotted patterns. 


\section{$3 \quad$ Avoiding a dotted pattern of length 2}

In this section, we present some enumeration results for permutations (and involutions) avoiding a dotted pattern of length 2, possibly along with one or more classical patterns. Considering the Wilf equivalences, we obviously obtain

$$
\left|S_{n}(2 \dot{1})\right|=\left|S_{n}(\dot{1})\right|=\left|S_{n}(\dot{1} 2)\right|=\left|S_{n}(\dot{2} 1)\right| .
$$

Moreover Lemma 1 induces $S_{n}(2 \dot{1})=S_{n}^{w}(\overline{2} 1)$ and with the definition of the weak avoidance we also obtain $S_{n}(2 \dot{1})=S_{n}^{w}(2 \overline{1})$.

Now let us give some useful definitions and notations. A fixed point of a permutation $\pi \in S_{n}$ is an integer $i \in[n]$ such that $\pi_{i}=i$. A permutation without fixed point will be called derangement, and the set of $n$-length derangements is denoted $D_{n}$. A strong fixed point of a permutation $\pi \in S_{n}$ is an integer $i \in[n]$ such that $\pi_{i}=i$ and $\pi_{j}<\pi_{i}<\pi_{k}$ for all $j, k$ verifying $j<i<k$. An involution of length $n$ is a permutation $\pi \in S_{n}$ such that $\pi^{2}=i d_{n}$. Let $I_{n}$ be the set of $n$-length involutions.

Theorem 1 The set $S_{n}(2 i)$ is the set of the permutations with no strong fixed point. Its generating function is known (see A052186, [14]) and given by $\frac{F(x)}{1+x F(x)}$ where $F(x)=$ $\sum_{n>=0} n ! x^{n}$

Proof. Let $\pi$ be an $n$-length permutation having a strong fixed point $i$. We have $\pi_{i}=i$ and $\pi_{j}<\pi_{i}<\pi_{k}$ for $j<i<k$. Consequently, the entry $i$ cannot be extended into a pattern 21 in $\pi$. Thus $\pi \notin S_{n}(2 \dot{1})$. Conversely, if $\pi$ contains an entry $\pi_{i}$ that cannot be extended into a pattern 21 then we necessarily have $\pi_{j}<\pi_{i}<\pi_{k}$ for $j<i<k$ which implies that $\pi_{i}=i$. Thus $\pi$ contains a strong fixed point $i$ which completes the proof.

Theorem 2 The set $S_{n}(2 \dot{1}, 1 \dot{2})$ is the set of the permutations $\pi$ with no strong fixed point and without $i$ such that $\pi_{i}=n-i+1$ and $\pi_{j}>\pi_{i}>\pi_{k}$ for $j<i<k$. We have $\left|S_{n}(2 \dot{1}, 1 \dot{2})\right|=2\left|S_{n}(2 \dot{1})\right|-n$ !

Proof. By symmetry a permutation avoiding $1 \dot{2}$ has no $i$ such that $\pi_{i}=n-i+1$ and $\pi_{j}>\pi_{i}>\pi_{k}$ for $j<i<k$. Thus there does not exist a permutation $\pi \in S_{n}, n \geq 2$, that contains both the patterns $2 \dot{1}$ and $1 \dot{2}$. So we have $\left|S_{n}(2 \dot{1}, 1 \dot{2})\right|=\left|S_{n}\right|-2\left(\left|S_{n}\right|-\left|S_{n}(2 \dot{1})\right|\right)=$ $2\left|S_{n}(2 \dot{1})\right|-n !$

\begin{tabular}{|c|c|c|}
\hline Pattern & Sequence & Sloane [14] \\
\hline$\{2 \dot{1}\},\{1 \dot{2}\},\{\dot{1} 2\},\{\dot{2} 1\}$ & no strong fixed point & $A 052186$ \\
\hline$\{2 \dot{1}, 1 \dot{2}\}$ & $2\left|S_{n}(2 \dot{1})\right|-n !$ & new \\
\hline
\end{tabular}

Table 1: Enumeration for the sets of permutations avoiding dotted patterns of length 2 


\section{$3.12 i$ and one classical pattern of length 3}

In this part we give exhaustive results about the cardinalities of the sets of permutations avoiding $2 \dot{i}$ and one classical pattern of length 3 . We also consider the sets of involutions avoiding these patterns.

Theorem 3 For $n \geq 3$, the set $S_{n}(2 \dot{1}, 123)$ is enumerated by $c_{n}-2$ (see A120304, [14]) where $c_{n}=\frac{1}{n+1}\left(\begin{array}{c}2 n \\ n\end{array}\right)$ is the nth Catalan number.

Proof. It is well known that $S_{n}(123)$ is enumerated by the $n$th Catalan number (see for instance [4]). Now if $\pi \in S_{n}(123)$ contains 21 then Theorem 1 implies that there exists a strong fixed point $i$ in $\pi$. There are only two possibilities: either $i=1$ and $\pi=1 n(n-1) \ldots 2$ or $i=n$ and $\pi=(n-1) \ldots 21 n$. Thus $\left|S_{n}(2 \dot{1}, 123)\right|=c_{n}-2$ for all $n \geq 3$.

Theorem 4 The set $S_{n}(2 \dot{1}, 321)$ is enumerated by the Fine numbers $f_{n}$ (see A00095\%, [14]) defined by $c_{n}=2 f_{n}+f_{n-1}$ where $f_{1}=0, f_{2}=1$ and $c_{n}$ is the $n$th Catalan number.

Proof. In [12], it is proved that the number of permutations $\pi \in S_{n}(321)$ with no fixed point is enumerated by the Fine numbers. Thus it suffices to verify that a permutation $\pi \in$ $S_{n}(321)$ with no strong fixed point does not contain any fixed point. For a contradiction, let us assume that $\pi$ contains a fixed point $\pi_{i}=i$. Theorem 1 shows that there exists $j \neq i$ such that at least one of the following conditions is true: (a) $j<i$ and $\pi_{j}>\pi_{i}$; (b) $j>i$ and $\pi_{j}<\pi_{i}$. For the case (a), this implies that there does not exist $\pi_{k}, k>i$ such that $\pi_{k}<\pi_{i}=i$. Therefore each value of the set $\{1,2, \ldots, i-1, j\}$ appears (in $\pi$ ) on the left of $\pi_{i}$ which contradicts the hypothesis $\pi_{i}=i$. The case (b) is obtained similarly. Thus $\left|S_{n}(2 \dot{1}, 321)\right|=\left|S_{n}(321) \cap D_{n}\right|$ where $D_{n}$ is the set of $n$-length permutations with no fixed point (derangements). By [12], we conclude that $S_{n}(2 \dot{1}, 321)$ is enumerated by the $n$th Fine number $f_{n}$.

Theorem 5 The set $S_{n}(2 \dot{1}, 231)$ is enumerated by the Fine numbers $f_{n}$ defined by $c_{n}=$ $2 f_{n}+f_{n-1}$ where $f_{1}=0, f_{2}=1$ (see A000957, [14]) and $c_{n}$ is the $n$th Catalan number.

Proof. We use the well known bijection $\varphi$ between $S_{n}(321)$ and $S_{n}(231)$ found by Simion and Schmidt [13] (see also Bóna [4], Lemma 4.3, p. 130). They consider the right-to-left minima of $\pi \in S_{n}(231)$; i.e., the entries $\pi_{i}$ of $\pi$ such that $\pi_{j} \geq \pi_{i}$ for all $j>i$. The bijection $\varphi$ keeps the right-to-left minima of $\pi \in S_{n}(231)$ fixed and to place all other entries in increasing order. For example, if $\pi=85214376 \in S_{8}(231)$ then the right-to-left minima are 1,3 and 6 (in boldface) and $\varphi(\pi)=24517386 \in S_{8}(321)$. As a strong fixed point also is a right-to-left minimum the bijection $\varphi$ preserves the strong fixed points. Thus $\varphi$ induces a bijection from $S_{n}(2 \dot{1}, 231)$ to $S_{n}(2 \dot{1}, 321)$. We conclude using Theorem 4 .

Theorem 6 The set $S_{n}(2 \dot{1}, 213)$ is enumerated by the sequence $c_{n}-c_{n-1}=\frac{3(2 n-2) !}{(n+1) !(n-2) !}$ (see A000245, [14]) where $c_{n}$ is the nth Catalan number. 
Proof. Let $\pi$ be a permutation in $S_{n}(21,213)$. As $\pi$ avoids $213, \pi$ can be decomposed as follows: $\pi=k \delta \gamma$ where $\delta$ (resp. $\gamma$ ) is a sequence made up of the values in $\{(k+1), \ldots, n\}$ (resp. values in $\{1,2, \ldots,(k-1)\}$ ) such that $\delta$ and $\gamma$ also avoid the pattern 213 (see Bóna [4], p. 132). Now since $\pi$ avoids $2 \dot{1}$ the first entry $k$ of $\pi$ must be different from 1. It is straightforward to see that this condition is also sufficient. Thus the cardinality of $S_{n}(2 \dot{1}, 213)$ is exactly $c_{n}-c_{n-1}$ where $c_{n}$ is the $n$th Catalan number. Thus we deduce $\left|S_{n}(2 \dot{1}, 213)\right|=\frac{3(2 n-2) !}{(n+1) !(n-2) !}$.

The Wilf equivalences applied to the above results allow us to obtain the enumeration for all sets of permutations avoiding $2 i$ with a classic pattern of length 3 :

\begin{tabular}{|c|c|c|}
\hline Pattern & Sequence & Sloane \\
\hline$\{2 \dot{1}, 321\},\{2 \dot{1}, 312\},\{2 \dot{1}, 231\}$ & Fine & $A 000957$ \\
\hline$\{2 \dot{1}, 132\},\{2 \dot{1}, 213\}$ & $c_{n}-c_{n-1}=\frac{3(2 n-2) !}{(n+1) !(n-2) !}$ & $A 000245$ \\
\hline$\{2 \dot{1}, 123\}$ & $c_{n}-2$ & $A 120304$ \\
\hline
\end{tabular}

Table 2: Enumeration for the sets of permutations avoiding $2 \dot{1}$ and one classical pattern of length 3

Now we present a similar study for the sets of involutions avoiding $2 \dot{1}$ and a classical pattern of length 3 . Recall that $I_{n}$ denotes the set of involutions of length $n$, i.e., the set of $n$-length permutations $\pi$ such that $\pi^{2}=i d_{n}$.

Theorem 7 The following statements hold:

i) For $n \geq 1,\left|I_{n}(2 \dot{1})\right|=d_{n}$ where $d_{n}$ is recursively defined by $d_{1}=0$ and for $n \geq 2$, $d_{n}=\left|I_{n}\right|-\sum_{r=1}^{n-1} d_{r} \cdot\left|I_{n-1-r}\right|$.

For instance $d_{n}=0,1,1,4,9,31,94,337,1185,4540$ for $n \leq 10$.

ii) For $n \geq 3,\left|I_{n}(2 \dot{1}, 123)\right|=\left(\begin{array}{c}n \\ \left\lfloor\frac{n}{2}\right\rfloor\end{array}\right)-2$.

iii) For $n \geq 3,\left|I_{n}(2 \dot{1}, 132)\right|=\left|I_{n}(2 \dot{1}, 213)\right|=\left(\begin{array}{c}n-1 \\ \left\lfloor\frac{n-2}{2}\right\rfloor\end{array}\right)$,

iv) For $n \geq 3,\left|I_{n}(2 \dot{1}, 231)\right|=\left|I_{n}(2 \dot{1}, 312)\right|=$ fib $_{n}$ where fib $b_{n}$ is the nth Fibonacci number defined by $f i b_{1}=0, f_{i} b_{2}=1$ and $f i b_{n}=f i b_{n-1}+f i b_{n-2}$ for $n \geq 3$.

v) For $n \geq 1,\left|I_{2 n}(2 \dot{1}, 321)\right|=c_{n}$ and $\left|I_{2 n+1}(2 \dot{1}, 321)\right|=0$ where $c_{n}$ is the $n$th Catalan number.

Proof. $i$ ) Let $\pi$ be an $n$-length involution that contains $2 \dot{1}$. By Theorem $1, \pi$ can be written $\pi=\delta k \gamma$ where $k \geq 2, \delta \in S_{k-1}(2 i)$ and $\gamma$ is a subsequence of elements in $\{k+1, \ldots, n\}$. As $\delta$ belongs to $S_{k-1}$ and $\pi$ is an involution, $\delta \in I_{k-1}(2 \dot{1})$. On the other hand $\gamma$ considered as a permutation in $S_{n-k}$ is necessarily an involution. Therefore the 
set of involutions that contain $2 \dot{1}$ is enumerated by $\sum_{k=2}^{n}\left|I_{k-1}(2 \dot{1})\right| \cdot\left|I_{n-k}\right|$ which induces that for $n \geq 2$,

$$
\left|I_{n}(2 \dot{1})\right|=\left|I_{n}\right|-\sum_{k=2}^{n}\left|I_{k-1}(2 \dot{1})\right| \cdot\left|I_{n-k}\right| .
$$

ii) It is straightforward to see that only two involutions containing $2 \dot{1}$ avoid 123 : $1 n(n-$ $1) \ldots 2$ and $(n-1) \ldots 21 n$. Thus for $n \geq 3$ we have

$$
\left|I_{n}(2 \dot{1}, 123)\right|=\left|I_{n}(123)\right|-2=\left(\begin{array}{c}
n \\
\left\lfloor\frac{n}{2}\right\rfloor
\end{array}\right)-2 .
$$

iii) An $n$-length permutation $\pi$ that contains $2 \dot{1}$ and avoids 132 can be written $\pi=$ $\delta k(k+1) \ldots n$ where $k \geq 2, \delta \in I_{k-1}(2 \dot{1})$. So if $a_{n}=\left|I_{n}(2 \dot{1}, 132)\right|$ then $a_{n}=\left|I_{n}(132)\right|-$ $1-\sum_{r=2}^{n-1} a_{r}=\left(\begin{array}{c}n \\ \left\lfloor\frac{n}{2}\right\rfloor\end{array}\right)-1-\sum_{r=2}^{n-1} a_{r}$ for $n \geq 3$ anchored by $a_{2}=1$. In order to prove the result we proceed by induction on $n \geq 3$. For $n=3$ then $a_{3}=\left(\begin{array}{c}3 \\ \left\lfloor\frac{3}{2}\right\rfloor\end{array}\right)-1-1=1$ thus the result holds. For $n \geq 3$ we have,

$$
a_{n}=\left(\begin{array}{c}
n \\
\left\lfloor\frac{n}{2}\right\rfloor
\end{array}\right)-1-\sum_{r=2}^{n-1} a_{r}=\left(\begin{array}{c}
n-1 \\
\left\lfloor\frac{n}{2}\right\rfloor
\end{array}\right)+\left(\begin{array}{c}
n-1 \\
\left\lfloor\frac{n}{2}\right\rfloor-1
\end{array}\right)-1-\left(\begin{array}{c}
n-2 \\
\left\lfloor\frac{n-3}{2}\right\rfloor
\end{array}\right)-\sum_{r=2}^{n-2} a_{r} .
$$

With the recurrence hypothesis and after considering the two cases $n$ odd/even, we deduce $a_{n}=\left(\begin{array}{c}n-1 \\ \left\lfloor\frac{n-2}{2}\right\rfloor\end{array}\right)$.

$i v)$ We set $a_{n}=\left|I_{n}(2 \dot{1}, 231)\right|$. Let $\pi$ be an involution of $I_{n}(2 \dot{1}, 231)$. As $\pi$ avoids $2 \dot{1}$, $\pi_{n}=n$ does not occur. Thus we distinguish two cases: (a) $\pi_{n}=n-1$ and $\pi_{n-1}=n$; and (b) $\pi_{n}<n-1$.

For the case (a), the permutation $\pi^{\prime} \in S_{n-2}$ obtained by deleting the two last entries of $\pi$ also is an involution in $I_{n-2}(2 \dot{1}, 231)$. Conversely, each permutation $\pi \in I_{n}(2 \dot{1}, 231)$ (verifying the condition (a)) can be obtained from a permutation $\pi^{\prime}$ as above.

For the case (b), let us assume $\pi_{k}=n$ with $k<n-1$. We consider $\pi^{\prime} \in S_{n-1}$ obtained by deleting the entry $\pi_{k}=n$ in $\pi$. As $\pi$ avoids $231, n-1$ is on the right of $n$ in $\pi$. Moreover, we necessarily have $\pi_{k+1}=n-1$ because otherwise the subsequence $(k+1) \pi_{n-1} \pi_{n}$ is a pattern 231. Iterating this process, $\pi$ and $\pi^{\prime}$ can be written $\pi=$ $\delta n(n-1) \ldots k$ and $\pi^{\prime}=\delta(n-1) \ldots k$ where $\delta \in I_{k-1}(2 \dot{1}, 231)$. This induces that $\pi^{\prime}$ is a permutation in $I_{n-1}(2 \dot{1}, 231)$. Conversely each permutation $\pi \in I_{n}(2 \dot{1}, 231)$ can be obtained from a permutation $\pi^{\prime} \in I_{n-1}(2 \dot{1}, 231)$ by inserting $n$ just after the entry $\pi_{k-1}^{\prime}$.

Therefore we deduce, for $n \geq 3$ that $a_{n}=a_{n-1}+a_{n-2}$ anchored by $a_{1}=0$ and $a_{2}=1$. This defines the Fibonacci sequence.

$v$ ) Let $\pi \in I_{2 n+1}(2 \dot{1}, 321)$. As the length of $\pi$ is odd, there exists a fixed point $\pi_{k}=k$. As $\pi$ avoids $2 \dot{1}$, there exists $j<k$ (or $j>k$ ) such that $\pi_{j}>\pi_{k}\left(\right.$ or $\pi_{j}<\pi_{k}$ ). In these two cases this means that there exists a pattern 321 that is $\pi_{j} k \pi_{\pi_{j}}$ (or $\pi_{\pi_{j}} k \pi_{j}$ ) which is a contradiction. Thus $\left|I_{2 n+1}(2 \dot{1}, 321)\right|=0$. 
Now let $\pi \in I_{2 n}(2 \dot{1}, 321)$. In this case, we refer to a result of Deutsch, Robertson, and Saracino [6], which states that the number of 321-avoiding involutions on [2n] with no fixed points is $c_{n}$. Indeed, $\pi$ is $2 \dot{1}$-avoiding iff it has no fixed point (which follows from the 321-avoiding property and the fact that $\pi$ is an involution).

\begin{tabular}{|c|c|c|}
\hline Pattern & Sequence & Sloane \\
\hline $2 \dot{1}$ & $d_{1}=0$ and $d_{n}=\left|I_{n}\right|-\sum_{r=1}^{n-1} d_{r} \cdot\left|I_{n-1-r}\right|$ & new \\
\hline$\{2 \dot{1}, 123\}$ & $\left(\begin{array}{c}n \\
\left\lfloor\frac{n}{2}\right\rfloor\end{array}\right)-2$ & new \\
\hline$\{2 \dot{1}, 132\},\{2 \dot{1}, 213\}$ & $\left.\begin{array}{c}n-1 \\
\left\lfloor\frac{n-2}{2}\right\rfloor\end{array}\right)$ & $A 037952$ \\
\hline$\{2 \dot{1}, 231\},\{2 \dot{1}, 312\}$ & Fibonacci & $A 000045$ \\
\hline$\{2 \dot{1}, 321\}$ & $c_{\frac{n}{2}}$ if $n$ is even; and 0 otherwise & $A 126120$ \\
\hline
\end{tabular}

Table 3: Enumeration for the sets of involutions avoiding $2 i$ and one classical pattern of length 3

\section{$3.22 i$ and two classical patterns of length 3}

In this part we give exhaustive results about the cardinalities of the sets of permutations avoiding $2 \dot{1}$ and two classical patterns of length 3 . For $n \geq 5$, we have $\left|S_{n}(2 \dot{1}, 123,321)\right|=$ $\left|S_{n}(123,321)\right|=0$.

Theorem 8 The set $S_{n}(2 \dot{1}, 123,213)$ is enumerated by $2^{n-1}-1$ for $n \geq 2$ (see A000225).

Proof. It is well known that $S_{n}(123,213)$ is enumerated by $2^{n-1}$. Theorem 3 proves that $S_{n}(2 \dot{1}, 123)$ is the set $S_{n}(123)$ except the two permutations $\pi=1 n(n-1) \ldots 2$ and $\pi^{\prime}=(n-1) \ldots 21 n$. However only $\pi$ belongs to $S_{n}(213)$. Therefore we deduce $\left|S_{n}(2 \dot{1}, 123,213)\right|=\left|S_{n}(123,213)\right|-1=2^{n-1}-1$.

Theorem 9 The set $S_{n}(2 \dot{1}, 132,213)$ is enumerated by $2^{n-1}-1$ for $n \geq 2$ (see A000225).

Proof. Let $\pi$ be a permutation in $S_{n}(2 \dot{1}, 132,213)$. Using the proof of Theorem $6, \pi$ can be decomposed as follows: $\pi=k \delta \gamma$ where $k \neq 1, \delta$ (resp. $\gamma$ ) is a sequence made up of the values in $\{(k+1), \ldots, n\}$ (resp. $\{1,2, \ldots,(k-1)\})$ such that $\delta$ and $\gamma$ avoid the pattern 213. The fact that $\pi \in S_{n}(132)$ implies that $\delta \in S_{n}(132,213)$ and $\gamma$ does not contain any pattern 21. As $\left|S_{n}(132,213)\right|=2^{n-1}$, we deduce that the cardinality of $S_{n}(2 \dot{1}, 132,213)$ is exactly $\sum_{i=1}^{n-1} 2^{i-1}=2^{n-1}-1$.

Theorem 10 The set $S_{n}(2 \dot{1}, 123,231)$ is enumerated by $\frac{(n-1) n}{2}-1$ for $n \geq 3$ (see A000096). 
Proof. It is well known that $S_{n}(123,231)$ is enumerated by the polygonal number $\frac{(n-1) n}{2}+$ 1. Theorem 3 shows that $S_{n}(2 \dot{1}, 123)$ is the set $S_{n}(123)$ except the two permutations $\pi=1 n(n-1) \ldots 2$ and $\pi^{\prime}=(n-1) \ldots 21 n$. Therefore we deduce $\left|S_{n}(2 \dot{1}, 123,231)\right|=$ $\left|S_{n}(123,231)\right|-2=\frac{(n-1) n}{2}-1$.

Theorem 11 The set $S_{n}(2 \dot{1}, 321,132)$ is enumerated by $n-1$ for $n \geq 2$ (see A007953).

Proof. Let $\pi$ be a permutation in $S_{n}(2 \dot{1}, 321,132)$. As $\pi$ avoids $2 \dot{1}, \pi_{1} \neq 1$ and $\pi \neq 12 \ldots n$. Let us assume that $\pi_{i}=1$ for some $i \geq 2$. We have $\pi_{i-1}>\pi_{i}=1$. As $\pi$ avoids 321, $1<\pi_{1}<\pi_{2}<\ldots<\pi_{i-1}$ and $\pi_{i+1}<\pi_{i+2}<\ldots<\pi_{n}$. As $\pi$ avoids 132, the values $\pi_{1}, \ldots, \pi_{i-1}$ are necessarily consecutive. As $\pi$ avoids $2 \dot{1}, \pi$ does not contain any strong fixed point, which means that there does not exist any value greater than $\pi_{i-1}$ to the right of 1 in $\pi$. Thus $\pi_{i-1}=n$ and $\pi=k(k+1) \ldots n 12 \ldots(k-1)$ with $k \neq 1$. Therefore $\left|S_{n}(2 \dot{1}, 321,132)\right|=n-1$ for $n \geq 2$.

Theorem 12 The set $S_{n}(2 \dot{1}, 132,231)$ is enumerated by $2^{n-2}$ for $n \geq 2$ (see A034008).

Proof. Let $\pi$ be a permutation in $S_{n}(2 \dot{1}, 132,231)$. The case $\pi_{1} \neq n$ does not occur. To see this, assume for a contradiction that $\pi_{1}=k \leq n-1$. As $\pi$ avoids 231 and 132 we necessarily have $\pi_{n}=n$ which is a contradiction with the avoidance of $2 \dot{1}$. Thus we have $\pi_{1}=n$. Moreover if $\pi^{\prime}$ is the permutation in $S_{n-1}$ obtained from $\pi$ by deleting its first value $n$ then $\pi^{\prime}$ also belongs to $S_{n-1}(132,231)$. Conversely, if we add $n$ on the left from a permutation in $S_{n-1}(132,231)$, the obtained permutation obviously belongs to $S_{n}(2 \dot{1}, 132,231)$. Therefore we have $\left|S_{n}(2 \dot{1}, 132,231)\right|=\left|S_{n-1}(132,231)\right|=2^{n-2}$.

Theorem 13 The set $S_{n}(2 \dot{1}, 132,312)$ is enumerated by $2^{n-2}$ for $n \geq 2$ (see A034008).

Proof. This proof is similar to that of Theorem 12 since a permutation $\pi \in S_{n}(2 \dot{1}, 132,312)$ can be written $\pi=\pi^{\prime} 1$ where $\pi^{\prime}$ avoids 132 and 312. As $S_{n}(132,312)$ is enumerated by $2^{n-1}$, the set $S_{n}(2 \dot{1}, 132,312)$ also is.

Theorem 14 The set $S_{n}(2 \dot{1}, 321,312)$ is enumerated by the Fibonacci sequence defined by $f i b_{2}=f i b_{3}=1$ and $f i b_{n}=f i b_{n-1}+f i b_{n-2}$ for $n \geq 4$ (see A000045).

Proof. Let $\pi$ be a permutation in $S_{n}(2 \dot{1}, 321,312)$. As $\pi$ avoids $2 \dot{1}$ we have $\pi_{n} \neq n$. Moreover, $\pi$ avoids 312 and 321 implies that $\pi_{n-1}$ necessarily equals to $n$ and we also have either $\pi_{n-2}=n-1$ or $\pi_{n}=n-1$. If $\pi_{n}=n-1$ then the permutation $\pi^{\prime}$ obtained from $\pi$ by deleting the two last values also belongs to $S_{n-2}(2 \dot{1}, 321,312)$. On the other hand, if $\pi_{n-2}=n-1$ then the permutation $\pi^{\prime}$ obtained from $\pi$ by deleting $\pi_{n-1}=n$ belongs to $S_{n-1}(2 \dot{1}, 321,312)$. Conversely, each permutation $\pi \in S_{n}(2 \dot{1}, 321,312)$ can be obtained from a permutation $\pi^{\prime}$ as previous. Therefore, $\left|S_{n}(2 \dot{1}, 321,312)\right|=\left|S_{n-1}(2 \dot{1}, 321,312)\right|+$ $\left|S_{n-2}(2 \dot{1}, 321,312)\right|$ which defines the Fibonacci sequence.

Theorem 15 The set $S_{n}(21,312,231)$ is enumerated by the Fibonacci sequence defined by $f i b_{2}=f i b_{3}=1$ and $f i b_{n}=f i b_{n-1}+f i b_{n-2}$ for $n \geq 4$ (see A000045). 
Proof. Let $\pi$ be a permutation in $S_{n}(2 \dot{1}, 312,231)$. As $\pi$ avoids $2 \dot{1}$ we have $\pi_{n} \neq n$. Moreover, $\pi$ avoids 312 and 231 implies that there exists $i \geq 1$ such that $\pi_{i}=n$ and $\pi_{i+1}=n-1$. If $i=n-1$ then the permutation $\pi^{\prime}$ obtained from $\pi$ by deleting the two last value $n(n-1)$ also belongs to $S_{n-2}(2 \dot{1}, 312,231)$. On the other hand, if $i<n-1$ then the permutation $\pi^{\prime}$ obtained from $\pi$ by deleting $\pi_{n-1}=n$ belongs to $S_{n-1}(2 \dot{1}, 312,231)$. Conversely, each permutation $\pi \in S_{n}(21,321,312)$ can be obtained from a permutation $\pi^{\prime}$ as previous. Therefore, $\left|S_{n}(2 \dot{1}, 312,231)\right|=\left|S_{n-1}(2 \dot{1}, 312,231)\right|+\left|S_{n-2}(2 \dot{1}, 312,231)\right|$ which defines the Fibonacci sequence.

\begin{tabular}{|c|c|c|}
\hline Pattern & Sequence & Sloane \\
\hline$\{2 \dot{1}, 123,321\}$ & $0,1,2,4,0,0,0 \ldots$ & \\
\hline$\{2 \dot{1}, 123,132\},\{2 \dot{1}, 123,213\},\{2 \dot{1}, 132,213\}$ & $2^{n-1}-1$ & $A 000225$ \\
\hline$\{2 \dot{1}, 123,231\},\{2 \dot{1}, 123,312\}$ & $\frac{(n-1) n}{2}-1$ & $A 000096$ \\
\hline$\{2 \dot{1}, 132,231\},\{2 \dot{1}, 132,312\}$, & $2^{n-2}$ & $A 034008$ \\
$\{2 \dot{1}, 213,231\},\{2 \dot{1}, 213,312\}$ & & \\
\hline$\{2 \dot{1}, 132,321\},\{2 \dot{1}, 213,321\}$ & $n-1$ & $A 007953$ \\
\hline$\{2 \dot{1}, 231,312\},\{2 \dot{1}, 231,321\},\{2 \dot{1}, 312,321\}$ & Fibonacci & $A 000045$ \\
\hline
\end{tabular}

Table 4: Exhaustive results for $2 \dot{1}$ and two classical patterns of length 3.

\section{3 $2 \dot{1}$ and two classical patterns of length 3 or 4}

Below we present three results for permutations avoiding $2 \dot{1}$ and two patterns of length greater than two. We also present an open question for permutations avoiding the patterns $\{21,231,4123\}$.

Theorem 16 The set $S_{n}(2 \dot{1}, 231,4321)$ is enumerated by the Pell numbers (see A000129).

Proof. Let $\pi$ be a permutation in $S_{n}(21,231,4321)$. We necessarily have $n-2 \leq \pi_{n} \leq n-1$. Indeed, Theorem 1 implies that $\pi_{n} \neq n$; moreover if $\pi_{n} \leq n-3$ then (as $\pi$ avoids 231) all values in the interval $I=\left[\pi_{n}+1, n-1\right]$ appear in decreasing order to the right of $n$ in $\pi$. As $\pi$ avoids 4321, the set $I$ contains at most one element which produces a contradiction with $\pi_{n} \leq n-3$. Thus we have either $\pi_{n}=n-1$ or $\pi_{n}=n-2$.

Let $\pi^{\prime}$ be a permutation in $S_{n-1}(2 \dot{1}, 231,4321)$ and $\pi$ be the permutation in $S_{n}$ obtained from $\pi^{\prime}$ by increasing by one the entries $n-1$ and $n-2$ (resp. the entry $n-1$ ) and by appending $n-2$ (resp. $n-1)$ on the right. Then $\pi \in S_{n}(2 \dot{1}, 231,4321)$. Conversely, a permutation $\pi \in S_{n}(21,231,4321)$ such that $\pi_{n}=n-2\left(\right.$ resp. $\pi_{n}=n-1$ and $\left.\pi_{n-1} \neq n\right)$ can be obtained by the previous construction from a permutation $\pi^{\prime} \in S_{n-1}(2 \dot{1}, 231,4321)$. On the other hand, let $\pi^{\prime}$ be a permutation in $S_{n-2}(2 \dot{1}, 231,4321)$ and $\pi$ be the permutation in $S_{n}$ obtained from $\pi^{\prime}$ by appending the sequence $n(n-1)$ on the right. 
Then $\pi \in S_{n}(2 \dot{1}, 231,4321)$. Conversely, a permutation $\pi \in S_{n}(2 \dot{1}, 231,4321)$ such that $\pi_{n}=n-1$ and $\pi_{n-1}=n$ can be obtained from $\pi^{\prime} \in S_{n-2}(21,231,4321)$ by this construction. Therefore we have $\left|S_{n}(2 \dot{1}, 231,4321)\right|=2\left|S_{n-1}(2 \dot{1}, 231,4321)\right|+\left|S_{n-2}(2 \dot{1}, 231,4321)\right|$ anchored by $\left|S_{2}(2 \dot{1}, 231,4321)\right|=1$ and $\left|S_{3}(2 \dot{1}, 231,4321)\right|=2$. We obtain the Pell numbers (see A000129).

Theorem 17 The set $S_{n}(2 \dot{1}, 321,2143)$ is enumerated by $2^{n-1}-n+1$ (see A000325), i.e., an instance of the Eulerian numbers plus one.

Proof. Let $\pi$ be a permutation of $S_{n}(2 \dot{1}, 321,2143)$. We will say that $\pi$ has a descent in position $i \in[n]$ if $\pi_{i}>\pi_{i+1}$. We distinguish two cases: (a) $\pi$ contains only one descent; and (b) $\pi$ contains at least two descents (notice that $\pi$ cannot be the identity permutation since $i d_{n}$ does not avoid $\left.2 \dot{1}\right)$. First, we will enumerate $n$-length permutations verifying the condition (a). Let us assume that the only descent occurs at position $i \geq 1$. We necessarily have $\pi_{i+1}=1$. To see this, assume to the contrary that $\pi_{i+1} \geq 2$. As $\pi$ avoids 321 , the value 1 is on the left of $\pi_{i}$; as $\pi$ contains only one descent $\pi_{1}=1$ holds which contradicts that $\pi$ avoids 2i. Moreover, we have $\pi_{i}=n$. Otherwise, if $\pi_{i} \neq n$ then $n$ either is the last entry or just before a value smaller than it. This is a contradiction. The permutation $\pi$ can be written $\pi=\delta n 1 \gamma$. where $\delta$ (resp. $\gamma$ ) is an increasing subsequences of $\left[\pi_{1}, n-1\right]$ (resp. $[2, n-1]$ ). The cardinality of such permutations is $\sum_{i=1}^{n-1}\left(\begin{array}{l}n-2 \\ k-1\end{array}\right)=2^{n-2}$.

Now let us examine the case (b). The permutation $\pi$ contains at least two descents. Let $i$ be the position of the leftmost descent $\left(\pi_{i}>\pi_{i+1}\right)$. As $\pi$ avoids $2 \dot{1}$ we have $\pi_{1} \neq 1$ and $\pi_{n} \neq n$; as $\pi$ avoids 321 we have $\pi_{i+1}=1$; as $\pi$ avoids 2143, if $\pi_{j}>\pi_{j+1}$ for $j>i+1$ then $\pi_{j+1}<\pi_{1}$ and $\pi_{n}=\pi_{1}-1$. These conditions define a particular structure for $\pi$ : $\pi=k \ldots(k+i-1) 1 \delta n \ell \ldots k-1$ for some $\ell, 2 \leq \ell \leq k-1$. Therefore these permutations are enumerated by: $\sum_{k=3}^{n-1} \sum_{i=1}^{n-k}\left(\begin{array}{c}n-i-2 \\ k-3\end{array}\right)=2^{n-2}-n+1$. Thus $\left|S_{n}(2 \dot{1}, 321,2143)\right|=2^{n-1}-n+1$.

Theorem 18 The set $I_{n}(2 \dot{1}, 231,4321)$ is enumerated by the Padovan sequence (see A000931).

Proof. The proof of Theorem 16 shows the form of a permutation $\pi \in S_{n}(2 \dot{1}, 231,4321)$. Indeed, we have either $\pi=\delta n(n-1)$ or $\pi=\delta n(n-1)(n-2)$. We deduce $\left|I_{n}(2 \dot{1}, 231,4321)\right|=\left|I_{n-2}(2 \dot{1}, 231,4321)\right|+\left|I_{n-3}(2 \dot{1}, 231,4321)\right|$ anchored by $\left|I_{1}(2 \dot{1}, 231,4321)\right|=0$ and $\left|I_{2}(2 \dot{1}, 231,4321)\right|=1$ which gives the Padovan sequence (see A000931).

Now we present an open question about the Pisot sequence $a(n)$ defined by $a(4)=5$, $a(5)=11$ and $a(n+1)$ is the nearest integer to $\frac{a(n)^{2}}{a(n-1)}$.

Problem 1 The set $S_{n}(2 \dot{1}, 231,4123)$ is enumerated by a sequence $b(n)$ that takes values $5,11,24,52,113,246,536,1168$ for $4 \leq n \leq 11$. Is it true that $b(n)$ is the Pisot sequence for $n \geq 4$ (see A021008)? 


\begin{tabular}{|c|c|c|}
\hline Pattern & Sequence & Sloane \\
\hline$\{2 \dot{1}, 231,4321\}$ & Pell & $A 000129$ \\
\hline Involutions avoiding $\{2 \dot{1}, 231,4321\}$ & Padovan & $A 000931$ \\
\hline$\{2 \dot{1}, 321,2143\}$ & $2^{n-1}-n+1$ & $A 000325$ \\
\hline
\end{tabular}

Table 5: Permutations avoiding 21 and two classical patterns of length 3 or 4

\section{Avoiding a dotted pattern of length 3}

In this section we investigate the avoidance of one dotted pattern of length 3 , possibly along with other classical patterns. We obtain several results when we avoid one dotted pattern of length 3 and one or more classical patterns. Unfortunately, we could not determine the cardinality of $S_{n}(\dot{\tau})$ where $\dot{\tau}$ is a 3-length dotted pattern of the form $\tau_{1} \tau_{2} \dot{\tau_{3}}$. Thus we present three open problems:

Problem 2 The set $S_{n}(23 i)$ is enumerated by the sequence a $(n, 0)$ that takes values $1,1,2,5,17,71,357,2101$ for $n \geq 1$ (see A101897). Is it possible to prove that this sequence is recursively defined by: $a(n, 0)=\sum_{k=1}^{n}(-1)^{k-1} \cdot a(n, k)$ where $a(n, k)=\sum_{j=0}^{n-k} a(n-$ $k, j) \cdot a(j+k-1, k-1)$ for $0<k \leq n$ anchored by $a(0,0)=0$.

Problem 3 Is it possible to obtain the cardinalities of the sets $S_{n}(12 \dot{3})$ ? The first values are $1,1,2,7,35,218,1598$ for $n \geq 1$. This sequence does not appear in the Sloane Encyclopedia [14].

Problem 4 The set $S_{n}(13 \dot{2})$ is enumerated by the sequence $b(n)$ that takes values $1,1,2,4,13,58,299,180$ for $n \geq 1$. Is it possible to prove that this sequence is recursively defined by: $b(n+1)=a(n, 0)-b(n)$ where $a(n, 0)$ is defined in Problem 2?

\subsection{Avoiding $23 i$ and 321 - Catalan}

Before presenting the main results we provide two basic technical lemmas.

Lemma 4 Let $n \geq 1$ be an integer. A permutation $\pi$ belongs to $S_{n}(321)$ if and only if for every $i \geq 1$, we have $\pi_{i}=\min \left\{\pi_{i}, \pi_{i+1}, \ldots, \pi_{n}\right\}$ or $\pi_{i}=\max \left\{\pi_{1}, \pi_{2}, \ldots, \pi_{i}\right\}$.

Proof. Let $\pi$ be an $n$-length permutation avoiding 321 and $i$ be an integer such that $\pi_{i}>\pi_{i+1}$. Since $\pi$ avoids 321, it does not contain any value greater than $\pi_{i}$ before $\pi_{i}$. Thus $\pi_{i}=\max \left\{\pi_{1}, \pi_{2}, \ldots, \pi_{i}\right\}$. A similar argument gives $\pi_{i}=\min \left\{\pi_{i}, \pi_{i+1}, \ldots, \pi_{n}\right\}$ if $\pi_{i}<\pi_{i+1}$. Conversely, if $\pi$ contains the pattern 321 then there are $i<j<k$ such that $\pi_{i}>\pi_{j}>\pi_{k}$. Thus $\pi_{j}$ is neither the maximum of elements to its left and nor the minimum of elements to its right which gives the result. 
Lemma 5 Let $n \geq 1$ be an integer and $\pi$ be a permutation in $S_{n}(321)$. Then $\pi$ avoids 231 if and only if $\pi_{i} \neq i-1$ for $i \geq 2$.

Proof. Let $\pi \in S_{n}(23 i, 321)$. Let us assume that $\pi \neq i d_{n}$ avoids $23 \dot{1}$ and there exists $i \geq 2$ such that $\pi_{i}=i-1$. Lemma 4 states that either $\pi_{i}=i-1=\min \left\{\pi_{i}, \pi_{i+1}, \ldots, \pi_{n}\right\}$ or $\pi_{i}=i-1=\max \left\{\pi_{1}, \pi_{2}, \ldots, \pi_{i}\right\}$. The first case implies that there exists $j<i$ such that $\pi_{j}>i-1$. Therefore the subsequence $\pi_{j} \pi_{i}$ cannot be extended into the pattern 231 which is a contradiction. The second case does not occur since $i-1$ cannot be the maximum of $i$ positive integers. Conversely, if $\pi$ contains 23i, then there exists $i<j$ such that $\pi_{i}>\pi_{j}$ where the subsequence $\pi_{i} \pi_{j}$ cannot be extended in $\pi$ to a 231 pattern. As $\pi$ avoids 321, all entries before $\pi_{j}$ are smaller than $\pi_{j}$. Thus $\pi_{j} \geq j$ and there exists $j \geq 2$ such that $\pi_{j} \neq j-1$.

Theorem 19 The set $S_{n}(231,321)$ is enumerated by the $(n-1)$ th Catalan number $c_{n-1}=$ $\frac{1}{n}\left(\begin{array}{c}2(n-1) \\ n-1\end{array}\right)$ (see A000108).

Proof. Let $\pi$ be a permutation in $S_{n}(231,321)$. For $k \in[n+1] \backslash\{n\}$, we define the permutation $\delta \in S_{n+1}$ by: $\delta_{n+1}=k$, and $\delta_{i}=\pi_{i}+1$ if $\pi_{i} \geq k$, and $\delta_{i}=\pi_{i}$ otherwise.

It is straightforward to verify that if $k>\pi_{n}$ then $\delta \in S_{n+1}(231,321)$ (Lemma 5 excludes the case $k=n)$. We have the same remark when $\pi_{n}=n$ and $k \leq n-1$. Conversely, each permutation $\delta \in S_{n+1}(23 i, 321)$ can uniquely be obtained by this construction for some $k$ and $\pi \in S_{n}(23 \dot{1}, 321)$ with $k \neq n$. Indeed, Lemma 5 implies $\delta_{n+1} \neq n$. Now let us assume that there exists $i \leq n$ such that $\delta_{i}=i>\delta_{n+1}$. As $\delta$ avoids 321, we have $\delta_{j}<i$ for $j<i$. Thus the $(n+1-i)$ values $(i+1), \ldots,(n+1)$ are necessarily on the right of $\delta_{i}=i$, and by considering the value $\delta_{n+1}<i$, there are $(n+2-i)$ values on the right of $\delta_{i}$ which cannot be possible. Consequently, this case does not occur. The permutation $\pi$ verifies $\pi_{j} \neq j-1$ for $j \geq 2$. Lemma 5 implies that $\pi \in S_{n}(23 i, 321)$. Thus each permutation $\pi$ such that $\pi_{n}=k, k \leq n-2$ (resp. $k=n$ ), produces $(n-k)$ (resp. $n$ ) permutations of size $(n+1)$ verifying $\pi_{n+1}=k+1, \ldots, n-1, n+1$ (resp. $\left.\pi_{n+1}=1,2, \ldots, n-1, n+1\right)$. If we label by $(k)$ a permutation $\pi$ such that $\pi_{n}=n-k$ then such a permutation produces $k$ permutations of size $n+1$ labeled $(2),(3), \ldots,(k+1)$ and 12 is labeled $(2)$. This is the definition of succession rules for Catalan numbers (see the method ECO $[1,2]$ ).

Theorem 20 The set $S_{n}(231,321,213)$ is enumerated by the $(n-1)$ th central polygonal sequence $\left(\begin{array}{c}n-1 \\ 2\end{array}\right)+1$ (or Lazy Caterer's sequence, see A000124).

Proof. Let $\pi \in S_{n}(23 i, 321,213)$ and $\pi \neq i d_{n}$. We consider the smallest $i$ such that there exists $j>i$ verifying $\pi_{i}>\pi_{j}$. As $\pi$ avoids 321 and 213, we immediately have $\pi_{k}<\pi_{j}$ for $k<i$; $\pi_{k}>\pi_{i}$ for $i<k<j$; and $\pi_{j}<\pi_{k}<\pi_{i}$ for $k>j$. As $\pi$ avoids 23i, there exists at least one value between the entries $\pi_{i}$ and $\pi_{j}$, i.e., $i<j-1$. Conversely, each permutation verifying these properties belongs to $S_{n}(231,321,213)$. Therefore, if we also consider $i d_{n}$ then the cardinality of $S_{n}(23 \dot{1}, 321,213)$ is $\sum_{j=3}^{n} \sum_{i=1}^{j-2} 1=\left(\begin{array}{c}n-1 \\ 2\end{array}\right)+1$. 


\subsection{Avoiding $23 \dot{1}$ and 132 - Motzkin}

Theorem 21 The set $S_{n}(23 \dot{1}, 132)$ is enumerated by the $(n-1)$ th Motzkin number (see A001006).

Proof. Let $M_{n}$ be the set $S_{n}(231,132)$ and $m_{n}$ its cardinality. Let $\pi$ be a permutation in $M_{n}$. We write $\pi$ as follows: $\pi=\delta n \gamma$ where $\delta n$ and $\gamma$ are two subsequences of $[n]$ ( $\gamma$ is not empty). See Figure 1 for an illustration of the decomposition. As $\pi$ avoids 132, the set of values in $\delta n$ necessarily constitutes an interval $[s, n]$ for some $s \leq n$. Consequently, $\gamma$ is a permutation in $S_{s-1}$. As $\pi$ avoids $23 \dot{1}$ and $132, \gamma \in M_{s-1}$ and $\delta n$ can be viewed as a permutation in $M_{n-s+1}$. Conversely, such a permutation $\pi=\delta n \gamma$ verifying the above conditions on $\delta n$ and $\gamma$ belongs to $M_{n}$. We deduce that $m_{n}=\sum_{s=1}^{n-1} m_{n-s} m_{s-1}$ anchored by $m_{0}=m_{1}=1$.

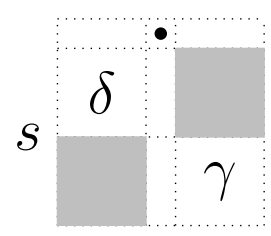

Figure 1: The special structure of a permutation in $S_{n}(23 \dot{1}, 132)$

\subsection{Avoiding 23i, 312 and 213 - Fibonacci}

Theorem 22 The set $S_{n}(231,312,213)$ is enumerated by the Fibonacci sequence (see A000045).

Proof. Let $\pi$ be a permutation in $S_{n}(231,312,213)$. As $\pi$ avoids 312 and 213 , we have either $\pi_{1}=1$ or $\pi_{n}=1$. If $\pi_{n}=1$ then the permutation $\pi^{\prime}$ obtained from $\pi$ by deleting $\pi_{n}=1$ and by decreasing by one all other values also belongs to $S_{n-1}(23 \dot{1}, 312,213)$. Moreover, as $\pi$ avoids $23 \dot{1}$ we cannot have $\pi_{n-1}^{\prime}=1$ (otherwise $\pi_{n-1}=2$ and $\pi_{n}=1$ contradicts that $\pi$ avoids 23i). Thus we have $\pi_{1}^{\prime}=1$ and thus $\pi_{1}=2$. Let $\pi^{\prime \prime}$ be the permutation obtained from $\pi^{\prime}$ by deleting the first entry 1 and by decreasing by one all other values, then $\pi^{\prime \prime} \in S_{n-2}(23 \dot{1}, 312,213)$. Conversely, if $\pi^{\prime \prime} \in S_{n-2}(23 \dot{1}, 312,213)$ then the permutation $\pi$ obtained from $\pi^{\prime \prime}$ by appending 2 (resp. 1) at the left (resp. at the right) then $\pi \in S_{n}(23 \dot{1}, 312,213)$. On the other hand, if $\pi_{1}=1$ then the permutation $\pi^{\prime}$ obtained from $\pi$ by deleting $\pi_{1}=1$ and by decreasing by one all other values also belongs to $S_{n-1}(23 \dot{1}, 312,213)$. Conversely, each permutation $\pi \in S_{n}(23 \dot{1}, 312,213)$ can be obtained from a permutation $\pi^{\prime} \in S_{n-1}(231,312,213)$ by adding the entry one on the left and by increasing by one all other values. Finally, if $a(n)=\left|S_{n}(231,312,213)\right|$ then $a(n)=a(n-1)+a(n-2)$ anchored by $a(1)=1, a(2)=1$. 


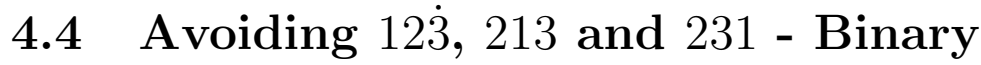

Theorem 23 The set $S_{n}(12 \dot{3}, 213,231)$ is enumerated by the binary sequence $2^{n-2}$ (see A034008).

Proof. Let $\pi$ be a permutation in $S_{n}(12 \dot{3}, 213,231)$. As $\pi$ avoids 213 and 231, such a permutation necessarily has: either $\pi_{i}=\max \left\{\pi_{1}, \ldots, \pi_{i}\right\}$ or $\pi_{i}=\max \left\{\pi_{1}, \ldots, \pi_{i}\right\}$. Thus we have $\pi_{1}=1$ or $\pi_{1}=n$. If $\pi_{1}=1$ then $\pi_{2}=2$ since $\pi$ avoids $12 \dot{3}$. Consequently each permutation $\pi$ can be written as either $\pi=n \pi^{\prime}$ or $\pi=12 \pi^{\prime \prime}$ where $\pi^{\prime}$ (resp. $\pi^{\prime \prime}$ ) belongs to $S_{n-1}(12 \dot{3}, 213,231)$ (resp. $\left.S_{n-2}(213,231)\right)$. Finally, by induction we obtain $\left|S_{n}(12 \dot{3}, 213,231)\right|=2^{n-3}+2^{n-3}=2^{n-2}$.

\subsection{Avoiding 213, 231 - Riordan}

Theorem 24 The cardinality of the set $S_{n}(\dot{2} 13,231)$ is given by the generating function $\frac{\left(1-z-\sqrt{1-2 z-3 z^{2}}\right) z}{1+z+\sqrt{1-2 z-3 z^{2}}}$ (see A005043 for the Riordan sequence).

Proof. It is well known that a permutation $\pi \in S_{n}(231)$ can be written $k \delta \gamma$ where $k \geq 1$, $\delta \in S_{k-1}(231)$ and $\gamma$ is a sequence of values in $\{k+1, \ldots, n\}$ that also avoids 231 . Such a permutation can be illustrated by a rooted binary tree $T$ with $n$ nodes as follows: $T$ is the rooted binary tree with its left subtree $T_{\ell}$ (resp. right subtree $T_{r}$ ) represents $\delta$ (resp. $\gamma$ considered as a permutation in $\left.S_{n-k}(231)\right)$. Using the classical pre-order bijection between binary trees and Dyck paths the permutation $\pi$ can be viewed as a Dyck path. See Figure 2 for an example of the correspondence between $\pi \in S_{n}(231)$, its binary tree with $n$ nodes, and its associated Dyck path.

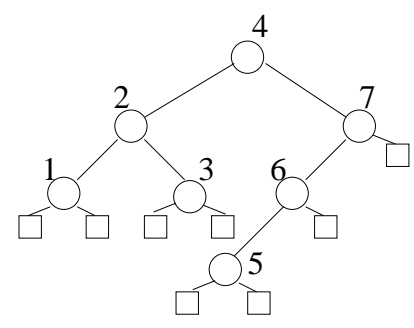

Figure 2: A permutation $\pi=4213765 \in S_{7}(\dot{2} 13,231)$ with its corresponding binary tree and the associated Dyck path $U U U D D U D D U U U D D D$ where $U=(1,1)$ and $D=$ $(1,-1)$.

As $\pi$ avoids 213 , the sequence $\gamma$ also avoids $\dot{2} 13 ; k \neq 1$ and $\delta$ and $\gamma$ are not empty. Moreover the avoidance of 213 implies that: (a) each subsequence $\pi_{i} \pi_{j}$ of $\pi$ with $\pi_{i}<\pi_{j}$ can be expanded into a 213 pattern; and (b) each subsequence $\pi_{i} \pi_{j}$ of $\pi$ with $\pi_{i}>\pi_{j}$ can be expanded into a 213 pattern. These two conditions also hold for the subsequence $\gamma$. Moreover the sequence $k \delta$ always verifies (b) since $\gamma$ contains the value $n$. Thus the 
permutation $\pi$ belongs to $S_{n}(\dot{2} 13,231)$ if and only if $\gamma$ avoids $\dot{2} 13$ and 231 , and $\delta$ verifies (a).

Now if we consider these conditions in the context of Dyck path (via the above bijection), the condition $\delta$ verifies (a) means that the Dyck path corresponding to the left subtree $T_{\ell}$ (of size at least 2) does not contain any occurrence of $U D U$. To see this, assume for a contradiction that $T_{\ell}$ contains an occurrence $U D U$. Then $\pi$ verifies $\pi_{i}=k$ and $\pi_{i+1}=k+1$ for some $i$ and $k$ such that $\pi_{j}>k$ if $j<i$. Thus the subsequence $k(k+1)$ cannot be expanded into a 213-pattern in $\pi$ which contradicts the condition $\delta$. So, these Dyck paths are enumerated by the Motzkin numbers (see [14], A001006). Therefore, the cardinalities of $S_{n}(\dot{2} 13,231)$ is given by the generating function: $A(z)=z^{2} M(z)(z+A(z))$ where $M(z)$ is the generating function of the Motzkin numbers. Thus we obtain

$$
A(z)=\frac{\left(1-z-\sqrt{1-2 z-3 z^{2}}\right) z}{1+z+\sqrt{1-2 z-3 z^{2}}},
$$

which is equal to $z(R(z)-1)$ where

$$
R(z)=\frac{2}{\left(1+z+\sqrt{1-2 z-3 z^{2}}\right)}
$$

is the well-known Riordan generating function.

From this previous proof, we directly deduce the following corollary.

Corollary 1 The cardinality of the set $S_{n}^{w}(\overline{2} 13) \cap S_{n}(231)$ is given by the Motzkin numbers (see A001006).

\subsection{Avoiding 23i, 2413 and 3142 - Catalan}

Theorem 25 The set $S_{n}(231,2413,3142)$ is enumerated by the $(n-1)$ th Catalan number.

Proof. Let $\pi$ be a permutation in $S_{n}(231,2413,3142)$. As $\pi$ avoids the two patterns 2413 and $3142, \pi$ is separable; i.e., $\pi$ can be written either (a) $\pi=\delta \gamma$ where $\delta \in S_{k}$ for some $k \leq n-1$, or (b) $\pi=\delta \gamma$ where $\gamma \in S_{k}$ for some $k \leq n-1$. For each case, we assume that $\delta$ is minimal in the sense that $\delta$ does not belong to the considered case. Moreover, $\pi$ avoids $23 \dot{i}$ implies that $\delta$ and $\gamma$ also avoid 23i; and for the case (b), $\delta$ is not reduced to one element. Thus the structure of permutations in the cases (a) or (b) induces the two following equalities.

Let $A(z)$ (resp. $B(z)$ ) be the generating function for permutations avoiding $23 \dot{1}$ and verifying (a) (resp. verifying (b)); let $F(z)=A(z)+B(z)+1$ the generating function for the set $S_{n}(23 \dot{1}, 2413,3142)$.

$$
\left\{\begin{array}{l}
A(z)=z F(z)+B(z)(F(z)-1) \text { and } \\
B(z)=(A(z)-z)(F(z)-1) .
\end{array}\right.
$$

If we sum these two equalities we obtain:

$$
F(z)^{2}-3 F(z)+z+2=0 .
$$


Thus we conclude $F(z)=\frac{3-\sqrt{1-4 z}}{2}$ which is exactly $1+z C(z)$ where $C(z)$ is the well-known Catalan sequence.

\begin{tabular}{|c|c|c|}
\hline Pattern & Sequence & Sloane \\
\hline$\{23 \dot{1}, 321\}$, & $c_{n-1}$ & $A 000108$ \\
\hline$\{23 \dot{1}, 321,213\}$ & $\left(\begin{array}{c}n-1 \\
2\end{array}\right)+1$ & $A 000124$ \\
\hline$\{23 \dot{1}, 132\}$ & Motzkin & $A 001006$ \\
\hline$\{23 \dot{1}, 312,213\},\{23 \dot{1}, 312,132\}$ & Fibonacci & $A 000045$ \\
\hline$\{31 \dot{2}, 132\}$ & Riordan & $A 005043$ \\
\hline$\{13 \dot{2}, 213,231\}$ & $2^{n-2}$ & $A 034008$ \\
\hline$\{23 \dot{1}, 2413,3142\}$, & $c_{n-1}$ & $A 000108$ \\
\hline
\end{tabular}

Table 6: Some results for a dotted pattern of length 3 and other classical patterns.

\section{Acknowledgements}

I would like thank the anonymous referee for their constructive comments.

\section{References}

[1] E. Barcucci, A. Del Lungo, E. Pergola and R. Pinzani. ECO: a methodology for the Enumeration of Combinatorial Objects. Journal of Difference Equations and Applications, 5:435-490, 1999.

[2] E. Barcucci, A. Del Lungo, E. Pergola and R. Pinzani. From Motzkin to Catalan permutations. Discrete Mathematics, 217:33-49, 2000.

[3] J.L. Baril and P.T. Do. ECO-generation for some restricted classes of compositions. Submitted, 2011.

[4] M. Bóna. Combinatorics of permutations. Chapman\&Hall, 2004, Chap. 4. 129-173.

[5] S. Butler and M. Bousquet-Melou. Forest-like permutations. Ann. Combin., 11:335$354,2007$.

[6] E. Deutsch, A. Robertson and D. Saracino. Refined restricted involutions. European Journal of Combinatorics, 28(1):481-498, 2007.

[7] O. Guibert. Combinatoires des permutations à motifs exclus en liaison avec mots, cartes planaires et tableaux de young. Thèse de l'université de Bordeaux I, 1996.

[8] S. Kitaev and T. Mansour. A survey on certain pattern problems. University of Kentucky research report 2003-09, available at http://www.ms.uky.edu/ math/MAreport/survey.ps. 
[9] D.E. Knuth. The art of computer programming. Vol. 1, Addison-Wesley, Reading, MA, 1973.

[10] T. Mansour. Permutations avoiding a pattern from $S_{k}$ and at least two patterns from $S_{3}$. Ars. Combin., 62:227-239, 2002.

[11] L. Pudwell. Enumeration schemes for permutations avoiding barred patterns. The electronic journal of combinatorics, 17, 2010.

[12] A. Robertson, D. Saracino and D. Zeilberger. Refined restricted permutations. Ann. Comb., 6:427-444, 2003.

[13] R. Simion and F.W. Schmidt. Restricted permutations. European Journal of Combinatorics, 6:383-406, 1985.

[14] N.J.A Sloane. On-Line Encyclopedia of Integer Sequences. Published electronically at http://www.research.att.com/ njas/sequences/.

[15] J. West. Permutations with forbidden subsequences and stack-sortable permutations. Ph.D. Thesis, MIT, 1990.

[16] J. West. Sorting twice through a stack. Theoretical Computer Sciences, 117:303-313, 1993.

[17] J. West. Generating trees and forbidden subsequences. Proceeding of sixth FPSAC, 441-450, 1994.

[18] J. West. Generating trees and the Catalan and Schröder numbers. Discrete Math., 146:247-262, 1995.

[19] A. Woo and A. Yong. "When is a Schubert variety Gorenstein?". Adv. Math., 207(1):205-220, 2006. 